\title{
Exceptive Constructions*
}

\author{
Kai von Fintel \\ University of Massachusetts at Amherst
}

\section{Introdiction}

Universally quantified statements exert an enormous attraction on language users. Their emotional force unfortunately clashes with the rather strict requirements on their truth. Small wonder then that there is a class of expressions used to reduce their force. These expressions typically diminish the domain quantified over. The exceptive constructions studied in this paper are prime examples. I will concentrate on the two types illustrated in (1), the highly grammaticized English but-phrase in (1a), and the 'free exceptive' with except for in (1b).

(1) a. Every student but John attended the meeting.

b. Except for John, every student attended the meeting.

This paper has two concerns, a narrowly descriptive one and a broader and potentially more interesting theoretical one. Although exceptive constructions and some of their core properties have been known since the times of the medieval semanticists, their proper analysis in a formal theory has proven very elusive. ${ }^{1}$ The analysis proposed in this paper will hopefully at least be more adequate than earlier attempts.

\footnotetext{
*Some of the material in this paper was presented at the Conference on CrossLinguistic Quantification at the LSA Summer Institute in Tucson, Arizona, July 22, 1989, at WCCFL X in Tempe, Arizona, March 1991, and at "Semantics and Linguistic Theory I", Cornell University, Ithaca, New York, April 1991. For comments and suggestions, I would like to thank especially Angelika Kratzer, Barbara Partee, Roger Higgins, Hotze Rullmann, Ginny Brennan, Paul Portner, Ed Keenan, Joe Moore, and Sue Tunstall. Research on this paper has been partially supported by the National Science Foundation under Grant No. BNS 87-19999 (Principal Investigators: Emmon Bach, Angelika Kratzer, and Barbara Partee). No one but myself should be blamed for the remaining mistakes and errors.

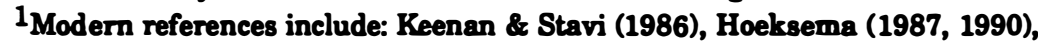
Landman \& Moerdijk (1979), Reinhart (1989).
} 
The broader concern of this paper, however, has to do with the notion of degrees of grammaticization and whether it can be captured in a compositional theory of the interaction between syntax and semantics. If the difference between the lexical items but and except for is only reconstructable by associating different kinds of lexical stipulations with them, this would be a disappointing result. A reduction to more general principles, on the other hand, would be a success. I will show that assuming something like Chierchia's three-layers hypothesis (1984), it can be derived that the free type of exceptive is less strong than the NP-internal type.

In Section 1 of this paper, I propose a semantic analysis of English but-phrases as in (2).

(2) a. Every student but John attended the meeting.

b. No student but John attended the meeting.

There is a problem of compositionality here: we would like to be able to give but a unified meaning that is applicable to both the positive determiner every and the negative one no. I show that it is indeed possible to give such a unified semantics, which turns out to have the added advantage of automatically explaining the co-occurrence restrictions of but-phrases. An interesting side-effect of the solution is that a previously unknown formal property is singled out that every and no share to the exclusion of all other basic determiners.

In Section 2, ways of connecting the semantics to the syntax are considered. The semantics of Section 1 puts certain constraints on possible syntactic analyses. I will argue for an NP-internal syntax of but-phrases and discuss two possible constituencies which merely correspond to different 'curryings' of the semantics.

Section 3 then is devoted to the analysis of free exceptives, especially those formed with except for as in (3).

(3) Except for the usual dissenters, most members didn't object.

There are at least two intriguing problems raised by this type of exceptive. How are free roaming exceptives associated with their target quantifier? I will side with Reinhart (1989) who argues that quantifier raising (QR) brings about the rendez-vous of free exceptive and target quantifier. The compositionality problem remains: how do exceptive and target combine after the association is established? I will suggest that the adjunct exceptive gets inside the target by binding a context set variable. The lower degree of grammaticization of free exceptives will be traced back to restrictions on the possible logical type of such free variables. 


\section{A Semantics for But-Phrases}

\subsection{What Do But'Phrases Mean?}

Considering the paradigm sentences with but in (2), repeated here, their truth-conditions are intutively rather obvious. In (2a), John is the only student who did not attend the meeting. In (2b), John is the only student who did attend the meeting. The sentences, if true, correspond to the diagrams in (4).

(2) a. Every student but John attended the meeting.

b. No student but John attended the meeting.

(4)

(2a)

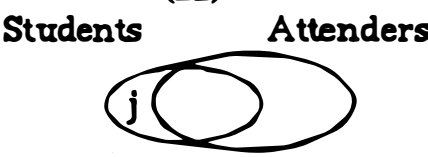

(2b)

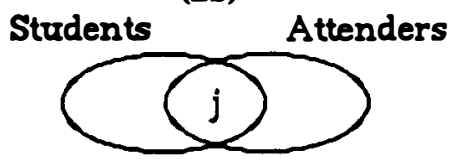

In trying to derive the truth-conditions of but-sentences compositionally, we would like the determiners every and no to have their usual denotation creating the generalized quantifiers given in (5).2

(5) 【every $\rrbracket(\llbracket$ student $\rrbracket)=(P \subseteq E \mid \bar{P} \cap \llbracket$ student $\rrbracket=\varnothing\}$

$$
\text { 【no】 }(\llbracket \text { student } \rrbracket)=\quad(P \subseteq E \mid P \cap \llbracket \text { student } \rrbracket=\varnothing)
$$

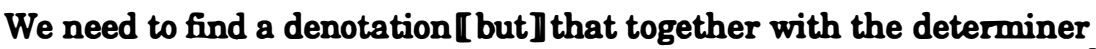
meanings in (5) derives the meanings given in (6) for the whole NP. ${ }^{3}$

(6) 【every student but John】 $=(P \subseteq E \mid \bar{P} \cap \llbracket$ student $\rrbracket=(\llbracket J o h n \rrbracket)\}$

$$
\text { 【no student but John } \rrbracket=\quad(P \subseteq E \mid P \cap \llbracket \text { student } \rrbracket=\{[J o h n \rrbracket)\}
$$

\footnotetext{
2The principal references on generalized quantifiers are Barwise \& Cooper (1981) and Keenan \& Stavi (1986). Throughout this paper, I will employ the notational framework of Barwise \& Cooper instead of Keenan's Boolean semantics or the lambda-expressions of Montague semantics. Everything here should be straightforwardly translatable into the other frameworks. [every] is defined in (5) in a way different from but equivalent to the standard formulation ([student] $\subset P$ ); the definition given here has the advantage of making the fundamental similarity between every and no more obvious.

3Te meanings in (6) are exactly those given by Keenan \& Stavi (1986) in their brief mention of exceptives. They do not however attempt a compositional derivation, which is my main concern here.
} 
The meanings in (6) are the result we want because they are equivalent to the intuitive meaning given for (1) above. What does [but] have to be for us to get the results in (6)? We especially want to have a unified meaning for but that can combine with both every and no without sneaking in a disjunctive stipulation. In the following sections, I will go through three successively stronger analyses that ultimately culminate in such an adequate semantics for but.

\subsection{But as a Minus Sign: Domain Subtraction}

The intuition which I will found my treatment of exceptives on is that they subtract entities from the domain of a quantifier. In a first approximation then, we could treat but as creating a noun modifier with a semantics as in (7). Some discussion of such an analysis can be found in Hoeksema (1987).

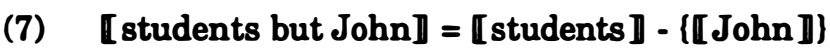

Our test sentence (2a) would then be true iff everyone who is a student but who isn't John attended the meeting. While I do think that domain subtraction as in (7) is the central part of the meaning of exceptive, as a semantics for but it fails on two grounds.

First, it fails to capture the co-occurrence restrictions of but-phrases. If but is a mere minus sign then the resulting set will be just like any other set without any distinguishing properties. The set of students minus John is a maximally dull set as far as set theory is concerned. There would then be no reason why some or most should not combine with it to form a well-formed noun phrase. But, *some students but John and *most students but John are clearly illformed.

Secondly, analyzing but as a minus sign allows some inferences to go through that are plainly illicit. The reason for this is that the universal determiners every and no are left downward monotone on their first argument as defined in (8).

(8) Left Downward Monotonicity ( $\downarrow$ mon) 4

$$
P \in D(A) \text { and } B \subseteq A \Rightarrow P \in D(B) \text {. }
$$

This property of universal determiners is what makes the inferences in (9) valid.

(9)

Every human being is mortal.

$\Rightarrow \quad$ Every male human being is mortal.

\footnotetext{
4Barwise \& Cooper (1981) use the term 'anti-persistent'.
} 


$$
\Rightarrow \quad \text { No human being is mortal. }
$$

If we put the left downward monotonicity of the universal determiners together with the view that but is a mere minus sign we now predict the inferences in (10) to be valid without further assumptions. The reason is that the set of students minus John and Jill is of course a subset of the set of students just minus John. Hence we should be able to infer down from the latter to the former.

Every student but John attended the meeting.

$? \Rightarrow$ Every student but John and Jill attended the meeting.

No student but John attended the meeting.

$? \Rightarrow$ No student but John and Jill attended the meeting.

But the inferences in (10) are blatantly incorrect. The conclusions imply that Jill is a student who did or did not attend the meeting. But that is something which we cannot validly infer from the premise. A satisfactory treatment of but then has to make sure that the downward monotonicity of the universal determiners is blocked in some way to prevent the inferences in (10) from falsely going through.

These problems notwithstanding, I would like to maintain the initial intuition as far as possible. I will assume that the central part of the meaning of but is indeed set subtraction. The strategy I pursue will be to add enough further conditions to the right hand side of the implication arrow in (11) to make it an equivalence. 5

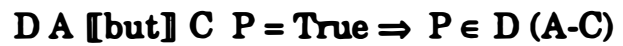

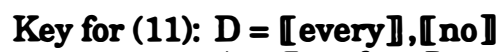

$$
\begin{aligned}
& A=\llbracket \text { student } \rrbracket \\
& C=\{[J o h n]\} \\
& \mathbf{P}=\llbracket \text { attended the meeting】 }
\end{aligned}
$$

At this point, I will not as yet commit myself to a particular bracketing of the noun phrase. Its internal constituency, the question of where the exceptive exactly operates, is the topic of Section 2.

5 For the semantics developed here to work, the NP after but has to introduce a set that can be subtracted from the set denoted by the head noun. This is arguably a healthy consequence because it appears that only NPs that can denote sets can appear after but; witness the ill-formedness of *all the students but each foreigner. 


\subsection{The Uniqueness Condition}

We have to strengthen the conditions even further. What does it mean to be the set of exception to a quantified statement? My answer is given in (12).

(12) The set of exceptions to a quantified sentence $D(A) P$ is the smallest set $C$ such that $D(A-C) P$ is true.

The exception set $C$ has to be the smallest set such that if it is subtracted from the quantifier domain the quantification comes out true. This can be factored out into two conditions, one of which is the domain subtraction clause we already know, and the other is essentially a condition of uniqueness. The formulation in (13) contains three equivalent ways of conceiving of the uniqueness condition.

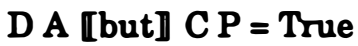

$$
\begin{aligned}
& \Leftrightarrow P \in D(A-C) \& \forall Y(P \in D(A-Y) \Rightarrow C \subseteq Y) \text {. } \\
& \Leftrightarrow P \in D(A-C) \& \forall B(B \subseteq A \& P \in D(B) \Rightarrow C \cap B=\varnothing) \\
& \Leftrightarrow P \in D(A-C) \& \cap\{Y \mid P \in D(A-Y)\}=C \\
& \Uparrow \\
& \text { Domain Uniqueness Condition }
\end{aligned}
$$

Consider again the paradigm sentence (1a) and the illustration of the state of affairs described by it, repeated here in (14). (13) then says that (1a) will be true iff everyone who is a student but who isn't John attended the meeting and it is the case that all the subsets of the students that contain only attenders do not contain John (that is a paraphrase of the second formulation of uniqueness). Note that this corresponds faithfully to the picture in (14). What the uniqueness condition boils down to then is that a but-phrase names the set responsible for the falsehood of the modified quantification.

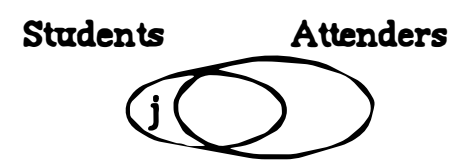

It should be obvious that the uniqueness condition is pragmatically natural. It ensures maximal relevance of the but-phrase: the exceptive not only is necessary to save the quantification, it also is the most economic way of doing that. The lexical meaning of but then has, I claim, internalized this pragmatically natural condition. 
Given the semantics for but in (13), the truth-conditions for exceptive sentences can be calculated as illustrated in (15). Apart from the application of the assumed standard definitions of 【every】 and [no】, all the steps in these derivations are justified by fairly elementary set-theoretic equivalences. 6 The resulting truth-conditions are exactly the ones that we set out to obtain. So far then, (13) is what we wanted. The test sentences get their desired meaning: (1a) is true iff John is the only student who did not attend the meeting, (1b) is true iff he is the only student who did attend the meeting.

(15) 【every】 A [but】 C P = True

$$
\begin{aligned}
& \Leftrightarrow P \in \llbracket \text { [every】 }(A-C) \& \forall Y(P \in \llbracket \text { every】 }(A-Y) \Rightarrow C \subseteq Y) \\
& \Leftrightarrow(A-C) \subseteq P \& \forall Y((A-Y \subseteq P \Rightarrow C \subseteq Y) \\
& \Leftrightarrow \bar{P} \cap A \subseteq C \& \forall Y(\bar{P} \cap A \subseteq Y \Rightarrow C \subseteq Y) \\
& \Leftrightarrow \bar{P} \cap A \subseteq C \& C \subseteq \bar{P} \cap A \\
& \Leftrightarrow \bar{P} \cap A=C
\end{aligned}
$$

$$
\begin{aligned}
& \llbracket n o \rrbracket A \llbracket b u t \rrbracket C P=T r u e \\
& \Leftrightarrow P \in \llbracket n o \rrbracket(A-C) \& \forall Y(P \in \llbracket n o \rrbracket(A-Y) \Rightarrow C \subseteq Y) \\
& \Leftrightarrow P \cap(A-C)=\varnothing \& \forall Y(P \cap(A-Y)=\varnothing \Rightarrow C \subseteq Y) \\
& \Leftrightarrow P \cap A \subseteq C \& \forall Y(P \cap A \subseteq Y \Rightarrow C \subseteq Y) \\
& \Leftrightarrow P \cap A \subseteq C \& C \subseteq P \cap A \\
& \Leftrightarrow P \cap A=C
\end{aligned}
$$

It is also easy to show that the unwanted inferences discussed earlier do not go through anymore. Consider the sentences in (16).

Assuming that (16a) is true, we can easily see that the semantics in (13) predicts that (16b) cannot be true at the same time. For if (16b) were true then all the sets of attending students would contain neither John nor Jill. But (16a) asserts that the set of students minus just John is a set of attenders, but it does contain Jill, in contradiction to (16b). The inference is therefore blocked.

(16) a. Every student but John attended the meeting.

b. Every student but John and Jill attended the meeting.

To complete the demonstration that (13) is a successful semantics for but, it has to be shown that the co-occurrence restrictions are suc-

6The set-theoretic tautologies employed are:
(i) $\mathrm{X}-\mathrm{Y} \subseteq \mathrm{Z} \Leftrightarrow \mathrm{X} \cap \overline{\mathrm{Z}} \subseteq \mathrm{Y}$
(ii) $\forall Y(X \subseteq Y \Rightarrow Z \subseteq Y) \Leftrightarrow Z \subseteq X$
(iii) $X \subseteq Y \& Y \subseteq X \Leftrightarrow X=Y$ 
cessfully captured. The crucial observation is that among simple natural determiners the existence of a unique exception set is only guaranteed by the universal ones, e.g., all (and its synonyms) and no (and its synonyms).

One direction is fairly elementary: if you have a universal determiner and it turns out that $P \notin D(A)$, then the unique exception is easy to obtain. If $D$ is no, then $P \cap A$ is the culprit, it should have been empty but wasn't; it is the unique exception we are looking for. If $D$ is all, then $\bar{P} \cap A$ is the offender and the unique exception, it should have been empty.

As an example of a determiner that is not universal and does not give rise to unique exceptions let us examine most. Consider the situation illustrated in (18) where (17a) is false because there a three students (Tom, John, and Harry) who did not attend the meeting while only two students (Bill and Mary) attended.

(17) a. Most students attended the meeting.

b. *Most students but Tom and John attended the meeting.

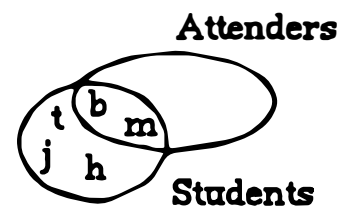

We could try to make the most-quantification true by excluding a sufficient number of non-attending students from the set we are quantifying over. So, we attempt (17b), excluding Tom and John, thus creating a situation where still only two students attended (Bill and Mary) but only one non-attender (Harry) remains. So now a majority of the students under consideration did attend the meeting. But note that since the most-claim is not a universal one we did not have to exclude all non-attending students. Hence we had a choice of which students to exclude. We could equally as well have excluded Tom and Harry or John and Harry. There is then obviously no unique set of students that we have to exclude. The uniqueness condition encoded in (13) brandmarks (17b) as false, since Tom and John are not the unique exception set to the quantification in (17a). Parallel thought experiments can be carried out for all determiners that even stand a chance of having exceptions (recall that the upward monotone ones are excluded by even more elementary considerations).

It has to be noticed that there are limiting cases where even a most-quantification has a unique exception. If there were only two students John and Harry and only one of them, Harry, attended the 
meeting, we can make the statement Most students attended the meeting true by excepting the unique student who did not attend the meeting: John. The existence of such exotic situations is obviously not enough for but to be able to occur with most.

The analysis of the co-occurrence restrictions of but that I propose then is that they are a grammaticization of the semantic fact that only universal determiners guarantee the existence of a unique exception set. In some way this will have to be built into the lexical entry for but. This concludes the demonstration that (13) is an adequate specification of the meaning of exceptive but. I have shown that (13) predicts the correct truth-conditions, that unwarranted inferences induced by the monotonicity of universal determiners are blocked, and that the co-occurrence restrictions of but are straightforwardly explained.

\section{The Syntax of But'-Phraces}

So far I have been careful not to commit myself to a position of how exactly the elements in a noun phrase like every student but John combine semantically and how this is linked to a particular syntactic structure. This section is devoted to these issues.

\subsection{The NP-Internal Operator'But}

A close look at the semantics in (13), repeated here in one of its formulations, reveals that the but-phrase must have access to both the determiner $\mathrm{D}$ and its domain $\mathrm{A}$. $\mathrm{D}$ is applied more than once in (19) to different sets, and A is subtracted from at various points.

$$
\begin{aligned}
& D \text { A [but] } C P=\text { True } \\
& \qquad \Leftrightarrow P \in D(A-C) \& \forall Y(P \in D(A-Y) \Rightarrow C \subseteq Y) .
\end{aligned}
$$

The necessity of 'simultaneous access' then excludes two initially attractive implementations. It is first not possible to have the exceptive operate solely on the domain $\mathrm{A}$, which would have made it possible to treat it as a fairly ordinary common noun modifier. It is also not possible to compute the noun-phrase denotation D (A) first and have the exceptive then take the result as its argument, which would have corresponded to an analysis of but-phrases as NP modifiers. The semantics in (19) forces more exotic analyses.

Assuming binary branching in both syntax and semantics, we have to decide whether the but-phrase applies first to the determiner and then to the common noun or the other way round. That is, we 
have to decide between two different 'curryings'? of the function denoted by the but-phrase, given in (20). 8

(20) a.

NP

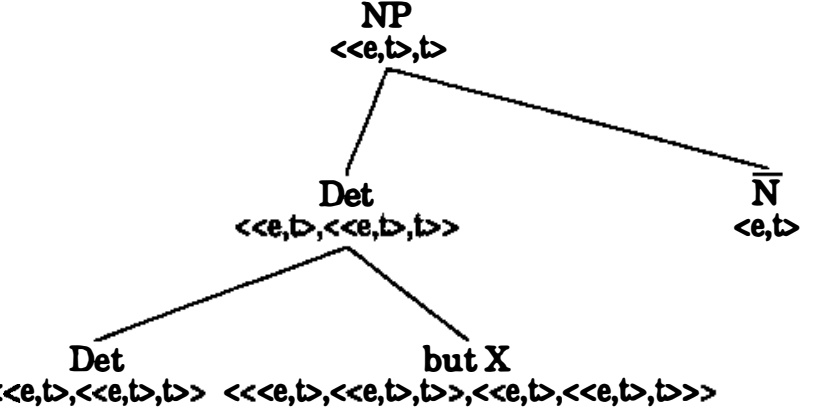

b.

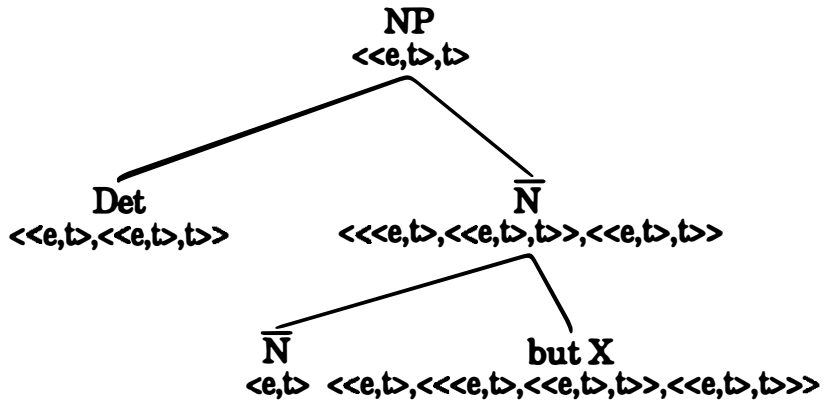

The first possibility is to treat but-phrases as modifiers of determiners which make them be of the same type as adverbs like almost in almost all (namely, functions from determiner denotations to determiner deenotations). This is actually implicit in an earlier proposal by Keenan \& Stavi (1986) who, however, do not give any compositional derivation but treat every...but... and no...but... as complex lexical items instead. Syntactically this would force us to accept either a discontinuous constituency or a local movement around the head noun. 9 Treating but-phrases as modifiers of determiners may

7Or rather 'schönfinkelizations', ef. Schönfinkel (1924).

8 Adopting the DP-hypothesis would of course mutliply the possible structures even more. At the moment I do not see any strong arguments for any particular structure.

9Such local wrappings are presumably independently motivated by constructions like an easy rug to clean or the first person we talked to (Ed Keenan, pc). For references on the status of discontinuity in the theory of grammar see the 
provide a natural connection to constructions like all but at most five students where we find a complex determiner phrase built with but to the left of the head noun.

The second and semantically more adventurous option has the but-phrase combine with the common noun first to give a higher type common noun which then takes the determiner as its argument, in a reversal of the ususal function-argument structure. This high type for common nouns is not usually employed in semantic analyses and we would like independent evidence for it. 10

It seems to me very difficult to decide between these options and in the absence of decisive evidence I will not commit myself to one of them in particular. It may even be that the data underdetermine the choice of structure, which would mean that there are two nearly equivalent grammars for this phenomenon.

What is crucial for the main argument of this paper is that the but-phrase, because of the built-in uniqueness condition, has to have a rather high logical type. In Section 3.4, I will suggest that this distinguishes them from the less grammaticized free exceptives.

\section{Free Erceptives}

Can the notion of degrees of grammaticization be reconstrued in a formal theory of grammar? That will be the focus of this section, in which I turn to the analysis of free exceptives with except for. The next subsections will establish that free exceptives are in some sense a less grammaticized cousin of but-phrases which at some point in their history presumably got 'subducted' from the status of an adjunct to the status of a logical operator inside the noun phrase. The main claim will be that the differences between the two types of exceptives follow from the fact that free exceptives have a lower logical type than the high one of but-phrases.

contributions in Huck \& Ojeda (1987) and Jim Blevins' dissertation (1990). See also Bach's (1979, 1981) Right Wrap operation which is one of the influences on the HPSG framework.

10There is one place in the literature I am aware of where this type is discussed. Partee \& Rooth (1983: 374fi) cite a manuscript by Robin Cooper where he proposes to analyze the reading of (i) where it means "most men swim and most women swim" by raising the type of the common noun phrase.

(i) Most men and women swim.

Partee \& Rooth discuss some of the issues that arise from admitting such type-raising. 


\subsection{Frcept for?}

What I call free exceptives are phrases marked with except for, which can appear both in left- and right-peripheral positions.

(21) a. Except for the famous detective, no one suspected the cook.

b. No one suspected the cook, except for the famous detective.

This positional freedom makes free exceptives crucially different from but-phrases. I will assume without much argument that it is not possible to consider free exceptives as being related to their associate quantifier by an s-structure movement rule. This is in accord with Baltin (1985) who argues that modifiers cannot in general be extraposed to the left. Suppose then that free exceptives are basegenerated as sentence adjuncts.

There are, I think, three degrees of semantic integration of the free exceptive into the sentence they modify. The loosest connection is found in cases where the exceptive is an afterthought, repair, or selfcorrection, illustrated by (22). It seems unlikely that these are amenable to a compositional analysis. I will leave them aside.

(22) Everyone loved the new show and noone thought it would be cancelled so soon. Except for George, of course.

An intriguing, perhaps best termed 'appositive', use is shown in (23). If near-universal determiners like most or few are employed, this probably gives rise to an implicature that it was not possible to use a universal determiner. In other words, they implicate the existence of an exception set. The sentences in (23) seem to have as their most prominent reading one where the exceptive gives further information about the exception set. (23a) then would convey that Joan is a notable exception to the generalization that cabinet members liked the proposal. It seems obvious that this appositive reading should only arise with free exceptives. They can, so to speak, 'wait' until the implicatures of the sentence are computed.

(23) a. Except for Joan, most cabinet members liked the proposal.

b. Except for John, few employees openly opposed the pay cut.

The third use of free exceptives, which is most similar to but-phrases, is the one I want to concentrate on here. In (24) the exceptive is used 'restrictively': only after the exceptive has done its thing will the quantification come out true. 
(24) a. Except for Jim, noone really liked the soup.

b. Except for Jane, my relatives are (all) total bores.

c. Except for the assistant professors, most faculty members supported the dean.

The account I will argue for again gives the exceptive a semantics of Domain Subtraction. The exceptive then has to 'get inside' the NP, which I show can be achieved via binding of a context set variable. Following work by Chierchia (1984), I will propose that such a variable can only be of type $\langle e, t\rangle$. As a consequence then, free exceptives will have a logically weaker semantics than but-phrases and can therefore also appear with non-universal determiners as (24c) shows.

\section{Reinhart (1989): Free Erceptives and QR}

Reinhart (1989) proposes that the association between free exceptive and target quantifier is established by quantifier raising (QR). The best evidence for this comes from examples where s-structure movement is routinely ruled out whereas $Q R$ is acknowledged to be possible. One such case is demonstrated in (25).11

(25) a. *Which cityi does [somebody from $t_{i}$ ] despise it?

b. Somebody from every city despises it.

c. Except for London, somebody from every city despises it.

d. *Somebody from every city despises it but London.

It was May $(1977,1985)$ who presented 'inverse linking' examples like (25b) to show that $Q R$ is freer than overt $w h$-movement which cannot extract from a complex subject as in (25a). (25c) shows that a free exceptive can associate with a quantifier inside a complex subject. As a tidbit and another argument for an s-structure extraposition movement for but-phrases, (25d) clearly shows that the limited possi-

11The discussion here is very much in the spirit of Reinhart's discussion. However, her own example (i) fails to make the point.

(i) Jokes about everyone were told except Felix. The biggest problem with (i) is that it sounds ungrammatical to most native speakers. Note that the underlying sentence itself, i.e. (iia), seems degraded, perhaps because extraposition of the PP as in (iib) is strongly preferred.

(ii) a. *? Jokes about everyone were told.

b. Jokes were told about everyone.

Furthermore, most of her examples have a right-peripheral exceptive formed with bare except (without for) and some of these do not sound as good as she claims. Except is perhaps more like but than she realizes (she actually does not discuss any differences between types of exceptives). To make sure that extraposition does not play a role, I use left-peripheral except for-phrases in my examples. 
bility of having but-phrases sentence-finally is subject to much stronger constraints than free exceptives.

Reinhart (1989) now proposes that after QR has applied to the associate of a free exceptive, the resulting configuration is in fact sufficient to allow the semantic rules to operate successfully. Depending on certain theoretical decisions that do not have to concern us here, we can give a sentence like (26a) one of the LF representations in (26b).

(26) a. Except for John, every student left.

b.

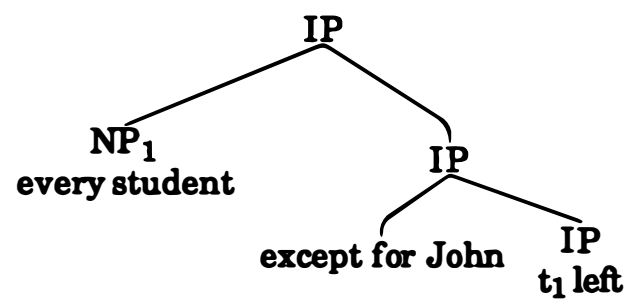

or

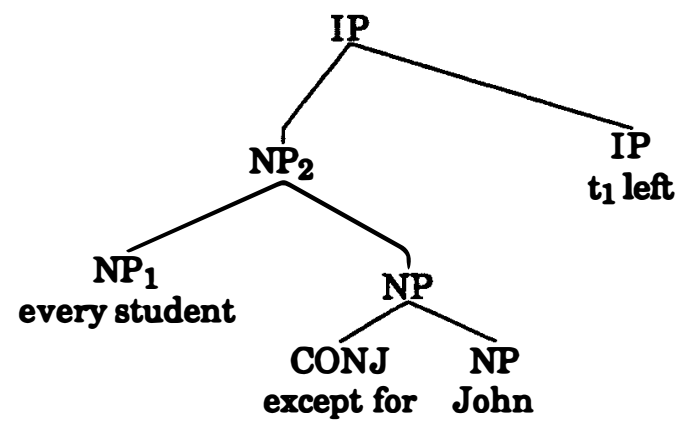

The choice between the two LFs in (26b) depends on whether in the second version the raised quantifier will be able to antecedent-govern its trace. Reinhart suggest that in an adjunction structure like this the antecendent-government definition may be fulfilled. She has to give the exceptive a very dubious structure, though, treating except for as a conjunction. We may then prefer the first version as less theoretically dicey.

As Reinhart herself realizes, even after the LFs in (26) have been created, it still remains unclear how they are interpreted correctly. First, the NP every student on its own is of course not the correct semantic binder of its trace. It seems obvious that it has to be the LFcreated constituent every student except for John that binds the argument trace in the IP. This problem will probably take care of 
itself once we have solved the second problem: how does the exceptive get to operate inside the associate quantifier although we only have an adjunction structure?

\section{Tricles of the Relative Clause Trade}

Let us suppose that we cannot touch the structural integrity of the associate quantifier, that is, that we cannot blow it up and reassemble the pieces as we need them. In some ways, this situation is reminiscent of the long standing debate about the constituency of relative clauses. 12 Here, semantic arguments favour an $\bar{N}$-modifier analysis, while there are other considerations that support an NP-modifier analysis. The last word about this recently rather dormant issue has presumably not been spoken. The study of exceptives can probably profit from some of the precedents set in the realm of relative clauses.

Robin Cooper in his dissertation (1975) proposed a way of interpreting correlative clauses (Hittite was his particular data source) that made them common noun modifiers semantically despite their fairly indisputable S-adjunct status. Bach and Cooper (1978)13 showed that this solution could also be used to reconcile an NP-level syntax of English relative clauses with an $\bar{N}$-semantics. ${ }^{14}$ The crucial technique is the introduction of a free variable at the $\overline{\mathrm{N}}$-level which can then later be filled in by the relative clause. In informal notation, the NP with relative clause in (27a) will be interpreted as in (27b).

a. [ [every man] who loves Mary]

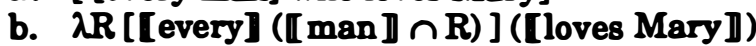

$\Leftrightarrow$ 【every】 (【man】 $\cap$ 【loves Mary】)

Non-trivial questions about the restrictiveness of the resulting framework arise then.15 What is the status of the NP-internal free variable posited here? One way of conceiving of the status of such free variables is that they are something like miraculously base-generated traces of base-generated adjuncts; base-generation would have to be less constrained than actual movement traces. For Hindi correlative clauses, Srivastav (1990) presents an alternative where what the correlative clause binds in its associate is not a free

\footnotetext{
12 A partial list of references should include at least: Stockwell, Schachter \& Partee (1973: Chapter 7 on 'Relativization') and their list of earlier references, Partee (1973, 1975), Chomsky (1975), McCawley (1981). Further references will be mentioned below.

13This article is an elaboration of Appendix $A$ of Cooper's dissertation.

14 An application to German relative clauses is given in von Stechow (1979).

15Some discussion can be found in Janssen (1983) and Partee (1984).
} 
variable ex nihilo but is in fact created by the demonstrative determiner of the NP.16 I favor a third possibility which was actually briefly put forward by Cooper himself (1975: 258f). When the free variable inside the noun phrase is not bound off by a relative clause, he suggests, it may represent the contextual restriction of the NP-interpretation to a specific restricted set of entities. 17 The need for such restrictions has been discussed in some recent work on generalized quantifiers (Westerstahl 1985, Johnsen 1987) and can be traced all the way back to early contributions to logical theory by Wallis, Boole, and de Morgan.

\subsection{Binding $\mathbf{R}$}

I would now like to urge that the analysis of free exceptives should be the one informally represented in (28).

(28) a. [IP [every student] $]_{i}$ [IP [except for John] [IP $t_{i}$ left ]]]

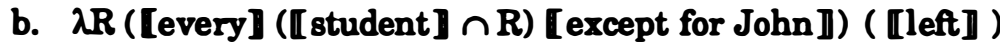

$\Leftrightarrow \llbracket$ every】 $(\llbracket \operatorname{man} \rrbracket \cap \overline{\mathrm{j}]}) \llbracket l e f t \rrbracket$

$\Leftrightarrow \llbracket \operatorname{man} \rrbracket-\hat{j}\} \subseteq \llbracket$ left $\rrbracket$.

A free variable $R$ of type <e,t> is introduced into the translation which is conjoined with the denotation of the common noun. The associate quantifier adjoins to the same IP that the free exceptive is a basegenerated adjunct of. Following Reinhart's idea the two phrases can then be interpreted as if they were sisters. The free exceptive gets quantified into the free variable $R$ inside the quantifier. The semantic effect is that of set subtraction.

Three obvious problems should immediately be addressed: (i) Why is this approach not also adequate for but-phrases? (ii) Does this approach explain why the co-occurrence restrictions of free exceptives are less rigid than those of but-phrases? and (iii) Why do sentences with universal determiners and free exceptives seem to have the same meaning as the corresponding sentences with but if all free exceptives do is set subtraction?

The important ingredient in answering all these questions is a restriction on the logical type of the context set variable. The particu-

\footnotetext{
16I hope to be able to discuss Srivastav's analysis and a possible different treatment some other time.

${ }^{17}$ Cooper refers to a similar suggestion made by Vendler (1967) who used an unexpressed relative clause to introduce the implict restrictions on definites. Another early reference is Hausser's dissertation (1974).
} 
lar formulation I adopt comes from work by Gennaro Chierchia. ${ }^{18}$ In his dissertation (1984: 74-90) he has some thoughts about the status of the hierarchy of logical types. There are, Chierchia says, basically three levels of natural language meanings: entities, properties, and functors. The third layer, the level of functors, is the exotic one. He proposes that there can be no variables of a functor type as formulated in (29). 19

\section{The "No Functor Anaphora Constraint" (Chierchia 1984)}

Functors do not enter anaphoric processes in natural languages.

Among the consequences of this constraint are the absence of wh-questions for determiners, non-predicative adverbs like almost, and other previously mysterious properties of natural language. For our context set variable the constraint will ensure that it can only be a set variable of type $<e, t>$. Remember that the uniqueness condition grammaticized in the lexical meaning of but forced us in Section 2 to give the but-phrase a functor type rather higher than $<e, t>$. The consequence of adopting (29) then is that an exceptive can only have the uniqueness condition as part of its lexical meaning if it is basegenerated in an NP-internal position. Free exceptives which get inside via binding of a variable cannot have the uniqueness condition as part of their lexical meaning. Since it was the uniqueness condition that explained the strict co-occurrence restrictions of butphrases, it is no surprise that free exceptives have looser restrictions and can indeed occur with non-universal determiners.

The weakness of the lexical meaning of free exceptives does not preclude that there are pragmatic strengthenings of that meaning. With universal determiners, the maximally relevant reading will

18Chierchia's ideas are inspired by Jespersen's hierarchy of primaries, secondaries, and tertiaries (1924: Chapter VII). I am very grateful to Paul Portner for reminding me of Chierchia's discussion.

19. The use of a free variable to stand in for the choice between a number of sentential connective meanings (because, in spite of, ...) by Stump $(1981,1985)$ in his treatment of the interpretation of free absolutes and adjuncts might be a countererample. I suspect though that his use of a free variable is rather different from the one discussed in this paper. His variable is not subject to wh-movement, anaphora, or binding. Still, this whole area needs a lot of further attention. For some comments on Stump's analysis see Partee (1984). Angelika Kratzer (pc) points out two further potential problems: only seems to be able to associate with focussed determiners or focussed bound variable pronouns, and functional questions (cf. Elisabeth Engdahl's work) seem to wh-move functors. At this point, I have no thoughts on these. 
still be the one where the exception stated is the unique smallest one. The perceived equivalence of universal statements modified by but-phrases or free exceptives then merely conceals the different way these readings come about.

\section{Conclusion}

The differences between the highly grammaticized but-phrases on the one hand and the somewhat looser free exceptives are then reconstructed as follows in my account.

Free exceptives are sentence adjuncts. Under their restrictive reading, they bind the context set variable in their intended target, which has been quantifier-raised to a position that makes it a sister of the exceptive. Since this is an anaphoric process, the type of the operation these exceptives denote can not be higher than $<e, t>$. Domain Subtraction is then all they can lexically mean. Depending on the quantificational force of the target, the meaning may be pragmatically strengthened to implicate uniqueness or minimality of the exception.

But-phrases are NP-internal operators. As such they are not subject to the constraint in (29). They incorporate the uniqueness condition into their lexical meaning, hence they are ungrammatical with non-universal associates. It is rather pleasant to speculate that but-phrases at some point may have been free exceptives just like except for-phrases are today. The process of grammaticization then would have sucked them down into the noun phrase (a generally active process in the history of languages that Roger Higgins has termed 'subduction'). Concomitantly to becoming an NP-internal operator, it could have acquired a stronger meaning incorporating the uniqueness condition. At this point, this is mere speculation, although I strongly suspect that something like this has to be what happened.

Let me conclude by saying that even if the specific decisions made in this paper turn out to be mistaken, the following four points might survive. (i) The study of exceptives and in general domain restrictors on quantifiers can serve as a probe into quantificational structures. (ii) Careful investigation of the techniques available to describe operators "from the outside" is still needed. (iii) Constraints such as the "No Functor Anaphora Constraint" of Chierchia deserve further attention. (iv) A formal reconstruction of the notion of grammaticization may not be impossible. 


\section{Refierences}

Abney, Steven Paul: 1987, The English Noun Phrase in its Sentential Aspect, Ph.D. Dissertation, MIT, Cambridge.

Bach, Emmon: 1979, 'Control in Montague Grammar', Linguistic Inquiry 10, 515-531.

-...: 1981, 'Discontinuous Constituents in Generalized Categorial Grammars', NELS 11.

--- and Robin Cooper: 1978, 'The NP-S Analysis of Relative Clauses and Compositional Semantics', Linguistics and Philosophy 2, 145-150.

Baltin, Mark: 1985, Toward a Theory of Movement Rules, Garland, New York.

Barwise, John and Robin H. Cooper: 1981, 'Generalized Quantifiers and Natural Language', Linguistics and Philosophy 4, 159-219.

van Benthem, Johan: 1984, 'Questions about Quantifiers', Journal of Symbolic Logic 49, 443-466.

Blevins, James P.: 1990, Syntactic Complexity: Evidence for Discontinuity and Multidomination, Ph.D. Dissertation, GLSA, University of Massachusetts at Amherst.

Chierchia, Gennaro: 1984, Topics in the Syntax and Semantics of Infinitives and Gerunds, Ph.D. Dissertation, GLSA, University of Massachusetts at Amherst.

Chomsky, Noam: 1975, 'Questions of Form and Interpretation', Linguistic Analysis 1, ???-??? [reprinted in Noam Chomsky, 1977, Essays on Form and Interpretation, North-Holland, Amsterdam, pp. 25-59].

Cooper, Robin H.: 1975, Montague's Semantic Theory and Transformational Syntax, Ph.D. Dissertation, GLSA, University of Massachusetts at Amherst.

Emonds, Joseph E.: 1985, A Unified Theory of Syntactic Categories, Foris, Dordrecht.

Hausser, Roland: 1974, Quantification in an Extended Montague Grammar, Ph.D. Dissertation, University of Texas, Austin.

Hoeksema, Jack: 1987, 'The Logic of Exception'. in A. Miller and J. Powers (eds), Proceedings of the Fourth Eastern States Conference on Linguistics (ESCOL 4), Ohio State University, Columbus, OH, pp. 100-113.

----: 1990, 'Exploring Exception Phrases', in Martin Stokhof and Leen Torenvliet (eds.), Proceedings of the Seventh Amsterdam Colloquium. 
Huck, Geoffrey J. and Almerindo E. Ojeda (eds.): 1987, Discontinuous Constituency (Syntax and Semantics, Vol. 20), Academic Press, Orlando.

Janssen, Theo M.V.: 1983, Foundations and Applications of Montague Grammar, Mathematisch Centrum, Amsterdam.

Jespersen, Otto: 1924, The Philosophy of Grammar, Allen \& Unwin, London.

Johnsen, Lars G.: 1987, 'There-Sentences and Generalized Quantifiers', in Peter Gärdenfors (ed.), Generalized Quantifiers: Linguistic and Logical Approaches, Reidel, Dordrecht, pp. 93-107.

Kadmon, Nirit and Fred Landman: 1990, 'Polarity Sensitive any and Free Choice any', in Martin Stokhof and Leen Torenvliet (eds.), Proceedings of the Seventh Amsterdam Colloquium.

Keenan, Edward L.: 1987, 'Multiply-Headed Noun Phrases', Linguistic Inquiry 18, 481-490.

--.- and J. Stavi: 1986, 'A Semantic Characterization of Natural Language Determiners', Linguistics and Philosophy 9, 253-326.

Landman, Fred and Ieke Moerdijk: 1979, 'Behalve als voorzetsel', Spektator: Tijdschrift voor Neerlandistiek 9, 335-347.

May, Robert: 1977, The Grammar of Quantification, Ph.D. Dissertation, MIT, Cambridge, (distributed by the Indiana University Linguistics Club, Bloomington).

-.-: 1985, Logical Form: Its Structure and Derivation, MTT Press, Cambridge.

McCawley, James D.: 1981, "The Syntax and Semantics of English Relative Clauses', Lingua 53, 99-149.

-.---: 1989, 'Individuation in and of Syntactic Structures', in Mark R. Baltin and Anthony S. Kroch (eds.), Alternative Conceptions of Phrase Structure, University of Chicago Press, Chicago, pp. 117138.

Partee, Barbara H.: 1973, 'Some Transformational Extensions of Montague Grammar', Journal of Philosophical Logic 2, 509-534 [reprinted in Barbara Partee (ed.), 1976, Montague Grammar, Academic Press, New York, pp. 51-76].

--.--: 1975, 'Montague Grammar and Transformational Grammar', Linguistic Inquiry 6, 203-300.

-.--:- 1984, 'Compositionality', in Fred Landman and Frank Veltman (eds.), Varieties of Formal Semantics, Foris, Dordrecht, pp. 283311.

---: 1988, 'Many Quantifiers', Proceedings of ESCOL 1988.

-.--:- 1989, 'Binding Implicit Variables in Quantified Contexts', Chicago Linguistics Society 25.

-... and Mats Rooth: 1983, 'Generalized Conjunction and Type Ambiguity', in in Rainer Bäuerle, Christoph Schwarze, and 
Amim von Stechow (eds.), Meaning, Use, and Interpretation of Language, de Gruyter, Berlin, pp. 361-383.

Reinhart, Tanya: 1989, 'Non-quantificational LF', to appear in A. Kasher (ed.), The Chomskyan Turn, Blackwell.

Rochemont, Michael S. and Peter W. Culicover: 1990, English Focus Constructions and the Theory of Grammar, Cambridge University Press, Cambridge [Cambridge Studies in Linguistics; 52].

Rooth, Mats Edward: 1985, Association with Focus, Ph.D. Dissertation, GLSA, University of Massachusetts at Amherst.

Schönfinkel, M.: 1924, 'Über die Bausteine der mathematischen Logik', Mathematische Annalen 92, 305-316.

Srivastav, Veneeta: 1991, 'The Syntax and Semantics of Correlatives', ms, to appear in Natural Language and Linguistic Theory.

von Stechow, Amim: 1979, 'Visiting German Relatives', in Rainer Bäuerle, Urs Egli, and Arnim von Stechow (eds.), Semantics from Different Points of View, Springer, Berlin, pp. 226-265.

Stockwell, Robert P., Paul Schachter, and Barbara Hall Partee: 1973, The Major Syntactic Structures of English, Holt, Rinehart, and Winston, New York.

Stump, Gregory T.: 1981, The Formal Semantics and Pragmatics of Free Adjuncts and Absolutes in English, Ph.D. Dissertation, Ohio State University.

-.--: 1985, The Semantic Variability of Absolute Constructions, Reidel, Dordrecht.

Vendler, Zeno: 1967, Linguistics in Philosophy, Ithaca, Cornell University Press.

Wells, R.: 1947, 'Immediate Constituents', Language 23, 81-117.

Westerståhl, Dag: 1985, 'Determiners and Context Sets', in Johan van Benthem and Alice ter Meulen (eds.), Generalized Quantifiers in Natural Language, Foris, Dordrecht, pp. 45-71.

Dept. of Linguistics

South College

University of Massachusetts

Amberst, MA 01003

fintel@linguist.umass.edu 